\title{
Circuit
}

Musiques contemporaines

\section{Entendu dans Cette ville étrange : quand on est dans la (marge) jusqu'au cou, il ne reste qu'à chanter}

\section{Marc Hyland}

Volume 27, numéro 1, 2017

URI : https://id.erudit.org/iderudit/1039674ar

DOI : https://doi.org/10.7202/1039674ar

Aller au sommaire du numéro

Éditeur(s)

Les Presses de l’Université de Montréal

ISSN

1183-1693 (imprimé)

1488-9692 (numérique)

Découvrir la revue

Citer ce document

Hyland, M. (2017). Entendu dans Cette ville étrange : quand on est dans la (marge) jusqu'au cou, il ne reste qu'à chanter. Circuit, 27(1), 71-76.

https://doi.org/10.7202/1039674ar d'utilisation que vous pouvez consulter en ligne. 


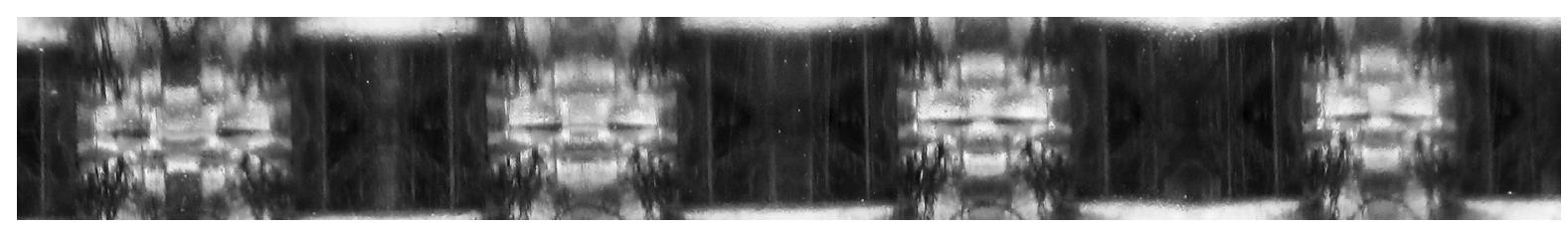

Photo: Christine Damme.

\section{ACTUALITÉS}

\section{Entendu dans Cette ville étrange ${ }^{1}$ : quand on est dans la (marge) jusqu'au cou, il ne reste qu'à chanter ${ }^{2}$}

Marc Hyland

\author{
No man is an island entire of itself; every man is a piece \\ of the continent, a part of the main; \\ if a clod be washed away by the sea, Europe is the less... \\ - John Donne, Extrait de MEDITATION XVII \\ Devotions upon Emergent Occasions, 1624.
}

En interdisant la musique et l'art, les talibans savent qu'ils occultent une portion de l'imaginaire collectif, sans doute pour minimiser l'évolution et l'actualisation de celui-ci, dans une crainte probable que les esprits ne se «dérèglent », n'en viennent à désirer autre chose que le statu quo. En bref, on pourrait dire que cette interdiction contribue à maintenir l'autre en marge du monde contemporain.

Dans une perspective inverse, celle du monde occidental «libre», où la censure esthétique opère rarement dans le monde de la création musicale, dans ce qui ressemble à l'indifférence lasse de qui aurait «tout entendu», reléguant le plus souvent à la musique populaire et aux arts visuels et scéniques les seuls chocs dignes de médiatisation, le compositeur se trouve-t-il lui aussi relégué dans quelque marge? La réponse semble évidente. Mais si sa musique se trouve en «marge », se pose-t-elle là de son gré ou s'y trouvet-elle acculée malgré elle?

Pour alléger ce texte (...), le «type spécifique» de compositeur auquel je réfère ici est celui ou celle qui compose de la «musique-de-concert-non-tonale-et-nonassimilable-au-vaisseau-de-la-pop-et-qu'on-ne-diffusequasiment-jamais-sauf-dans-de-rares-émissions-spécialisées-lorsqu'il-le-faut-pour-quelque-anniversaireet-dont-on-ne-parle-quasi-jamais-dans-les-médias-saufparfois-en-cas-de-décès-ou-d'événement-exceptionneldevant-être-évoqué-parce-qu'il-le-faut-bien ».

Qu'est-ce que la marge? On pense d'abord à l'espace blanc et vierge - Ô papier, sable chaud des intellects! - qui se trouve autour d'une page écrite ou imprimée, à ce qui s'oppose au centre, donc dans une périphé- 
rie et ainsi, par extension, à l'écart... Dans le jargon sociologique, la marginalisation est le fait, pour un individu ou un groupe d'individus, de s'écarter de la norme de la société, de s'en exclure ou d'en être exclu avec une rupture, parfois brutale, des liens sociaux. Coïncidence: le terme est d'un usage récent en sociologie, soit depuis la seconde moitié du $\mathrm{Xx}^{\mathrm{e}}$ siècle, plus ou moins l'époque à partir de laquelle la musique contemporaine s'est... marginalisée. Plus poétiquement, le terme est aussi utilisé en géologie, où la marge continentale désigne la partie immergée de la bordure d'un continent faisant le raccord avec les fonds océaniques. Dans cet esprit, la musique contemporaine serait, ou pourrait agir, comme raccord ou lien caché entre la culture musicale dominante (le continent) et la musique «inouie » (celle des abysses océaniques). Revoyons cette géologie à partir de son extrême: aux fonds océaniques correspondent les intervenants underground ou underwater, dont on n'entend nulle part la production, fruit d'artistes non enregistrés, non subventionnés, non recensés. Plus proches de la côte, immergés, se trouvent les compositeurs et les musiciens liés à ce qu'on appelle la musique contemporaine, dont la production relativement confidentielle par commandes, concerts et enregistrements est rendue possible par les arcanes de subventions étatiques (ou plus rarement privées), à peu près dégagée des contraintes du marché et des habituelles exigences de rentabilité. Le continent émerge ensuite progressivement des eaux pour laisser entendre et voir la myriade de déclinaisons de la musique mainstream, recevable et lisible par «tous», mais qui se voit de plus en plus appauvrie par les rouages impitoyables et la «gratuité » du Web, la musique rentable étant dorénavant réservée aux pics élevés des palmarès.

En divaguant maintenant à partir de la métaphore marine de Donne citée en incipit (écrite voilà quatre siècles), je dirais qu'à notre époque le compositeur large relatif du continent des musiques dominantes, duquel il se rapprocherait ou s'éloignerait, au gré des courants (!) et des jugements posés sur lui et sa manière personnelle de cultiver sa terre. (Les murs de quelque institution l'y protègent-ils du froid et du vent? Aura-t-il joint les rangs de quelque académie ou regroupement ou travaillera-t-il en solo, au rythme des possibilités que lui offre - ou laisse - le climat ambiant?)

Au fil du temps, certaines îles flottantes deviennent presqu'îles lorsque les œuvres de leurs auteurs parviennent à s'insérer dans l'oreille collective de façon assez soutenue et marquée, pénétrant ainsi l'inconscient. Une presqu'île est la partie saillante d'une côte, reliée à la terre par un isthme, ou serait-ce par la voie officielle d'un «isme»? Le minimal(isthme) des Américains Glass, Reich et autres Riley, par exemple, a fait de leurs œuvres des presqu'îles maintenant liées au continent de façon inéluctable, pour avoir su capter la «pulsation frénétique de l'Amérique moderne». Mais ce phénomène reste rare et le statut «flottant » du compositeur semble le plus répandu, sans que cela ne trahisse la vision de Donne. En dépit de tout, le compositeur ne vit pas, n'écrit pas en huis clos et ne saurait se suffire à lui-même. Il a besoin de la page du monde dont il fait partie, à un degré ou à un autre. Comme tout artiste, il est un migrant en quête d'amour.

Si on le compare aux pays d'où a émergé la tradition musicale occidentale, le Québec est-il différent quant à la place qu'il laisse aux compositeurs? Il faut comprendre que pour un français, un italien ou un allemand, la notion de tradition de musique classique est une évidence historique et tous reconnaitront que ces pays ont produit des figures marquantes attestant musicalement de leur réalité et de leur essence. Mais en termes d'Histoire, le Québec est encore adulescent et pour l'ensemble de la population, aucun compositeur d'ici n'a encore atteint le statut de figure consensuelle, réservé aux tenants et géants de la musique populaire que sont les Leclerc, Vigneault ou Ferland, pour ne 
nommer qu'eux, vecteurs indéniables d'une identité «québécoise». Ce qui importe, c’est que le Québec reste fertile dans cette verdeur, même si cette fertilité est le fruit d'une ambiguïté profonde. "Celui qui ne s'est pas encore nommé » a beaucoup à dire...

Est-ce naïveté ou nostalgie que de se demander s'il (pourra) existe(r) un son "musique contemporaine» qui représente le Québec dans toute sa complexité et sa nature profonde, dans lequel monsieur et madame tout le monde sauront se reconnaître et se retrouver comme ils le font dans une chanson de Ferland ou une pièce de Michel Tremblay? Cela est-il même souhaitable ou est-ce là un relent de nationalisme vieux style, quand les pays avaient des patrimoines aux «sonorités » typiques, avant que la mondialisation ne commence à tout quantizer, petit à petit, dans sa grande vague d'homogénéisation qui aplanira lentement mais sûrement les différences génétiques physiques et culturelles pour faire du monde un seul grand marché? En dépit de son jeune âge, il faudrait être de bien mauvaise foi ou sourd! - pour ne pas reconnaître que le Québec a produit des œuvres maîtresses qui sont là, figées sur le papier, en attente d'être ramenées à la vie pour nous parler. Lavenir verra-t-il - et voudra-t-il de - ce renouveau, à travers la quantité affolante d'œuvres existantes et de nouvelles œuvres créées, ou le puits du présent sera-t-il perpétuel, nous empêchant toute reconsidération du passé? Dans le déluge des données qui pleuvent sur tous, qui prendra le temps de réexaminer ainsi le passé?

Vieille rengaine: l'absence de véritable appareil critique ou réflexif concernant la création musicale québécoise est troublante, dans ce qui semble être une négation pure et simple de sa réalité. À ce sujet, j’ai retrouvé le passage suivant chez Milan Kundera, dans son ouvrage Les testaments trahis:

Je ne médirai jamais de la critique littéraire. Car rien n’est pire pour un écrivain que de se heurter à son absence. Je parle de la critique littéraire en tant que méditation, en tant qu'analyse [...]. Sans elle, toute œuvre est livrée aux jugements arbitraires et à l'oubli rapide. [...] La critique littéraire, imperceptiblement, innocemment, par la force des choses, par l'évolution de la société, de la presse, s'est transformée en une simple (souvent intelligente, toujours hâtive) information sur l'actualité littéraire 3 .

En substituant dans cet extrait le musical au littéraire et le compositeur à l'écrivain, on trouve un juste portrait de la situation actuelle, que les vingt années passées depuis la parution du livre n'ont pu qu'exacerber, ici et ailleurs. Si des musicologues - comme il s'en trouve déjà d'excellents, notamment en lien avec la présente revue -, mais aussi des sociologues, des philosophes ou autres esprits indépendants se penchaient sur la production musicale, non pour la qualifier de façon banale, mais pour tenter d'en dégager la teneur réelle, la pertinence relative, la signification, la direction et la présence dans la cité et sa culture, que trouveraient-ils? Le constat serait peut-être cruel, mais le fait qu'il provienne aussi de gens hors milieu pourrait induire un sentiment de réalité salutaire pour les compositeurs... À moins d'être «résolument modernes» et de confier cette tâche à des réseaux d'algorithmes?

Revenons à la page où vit le texte. Ses marges de blancheur - le blanc étant d'ailleurs en optique la somme de toutes les couleurs - ne procurent-elles pas une surface de ressourcement, sorte de plage ouverte propice à l'existence et à la lecture du texte qu'elles portent? Écrire de la musique contemporaine, sans même vouloir y distinguer quelque degré de hiérarchie ou de "difficulté», c'est inscrire quelque chose en marge du continent. Cette marge constitue un écosystème où coexistent nombre d'intervenants, outre les compositeurs, assez pour constituer un «milieu» à cette marge. Milieu duquel certains peuvent aussi se trouver ... en marge, comme la thématique de ce numéro de Circuit semble indiquer. En comparaison, la communauté des artistes visuels semble moins «invisible », la force de l'image aidant, et si le marché de l'art reste relativement confidentiel au Québec, les 
médias s'intéressent quand même à certaines figures, contribuant du coup à leur succès. Le cinéma d'auteur trouve aussi sa maigre pitance dans les médias, en dépit du fait qu'on estime son bassin cible à 3000 spectateurs, de façon générale.

$\mathrm{Si}$ on admet que toute ouvre est une demande d'amour et que l'artiste crée aussi pour combler un manque, que faire de l'indifférence du monde réel, hors milieu, devant cette création musicale «ni tonale ni techno »? Si tous les artistes du théâtre, du texte, de la danse, de la musique populaire, affirment depuis toujours être nourris et ne pouvoir évoluer qu'à travers et par l'amour qu'on leur porte, est-il possible que le compositeur reste perpétuellement sous-développé, en deçà de ses véritables capacités, de ce qu'il pourrait devenir s'il était entendu par et entendait lui-même l'écho du «vrai monde»? Peut-on imaginer ce qui adviendrait s'il recevait autre chose qu'un silence radio des non-spécialistes? Cette marge où il se voit confiné déterminerait-elle la couleur souvent sombre et dramatique, si ce n'est dépressive, de sa musique? (On jase...)

Si je me limite ici au Québec, il se trouve bien sûr des compositeurs qui ont été salués dans les médias et se trouvent maintenant plus près du «centre de la page». Claude Vivier semble ne pas avoir connu de purgatoire depuis son décès, son œuvre connaissant un engouement maintenant mondial et croissant. À quoi est-ce dû? Au caractère rituel profondément mélodique et humain de sa musique? À ses homophonies magiques? À sa «simplicité complexe»? Gilles Tremblay, par sa musique pétrie de nature, de poésie et de principes acoustiques fondamentaux, a lui aussi trouvé une résonance. Mais combien d'autres figures voient leurs productions ignorées de façon absolue depuis leur décès? Est-ce si grave, se demanderont certains? Si l'œuvre est forte, elle s'imposera? Patience alors, mais l'examen d'œuvres considérées « très fortes » au siècle passé, incluant celles de compositeurs européens, les trouvent encore bien... en marge.
Maintenant, l'avenir et un peu d'espoir. La technologie opère des changements, notamment dans la production de la musique, mais aussi dans sa promotion. Les capacités de dissémination de l'information offertes par des réseaux comme Facebook rendent la diffusion de concerts et d'ateliers beaucoup plus facile et transversale, au-delà des frontières et de contingences physiques. Cela paraît une évidence pour ceux qui sont nés avec, mais pour les plus de 45 ans, qui ont d'abord connu l'époque des concerts annoncés par communiqués postaux et téléphones, le nouveau paysage est formidablement dynamique et ouvert sur le monde entier. Cette diffusion s'opère maintenant à l'horizontale, moins soumise à la domination et la hiérarchie des organismes établis, ce qui pourrait aussi expliquer la fabuleuse prolifération des ensembles spécialisés à l'œuvre dans toutes sortes de lieux improbables, dans une attitude moins «attentiste». Le simple fait qu'un jeune compositeur puisse annoncer facilement et sans frais en ligne la création d'une œuvre à ses centaines d' 'amis » de par le monde démocratise dans les faits cette information, qui à une autre époque serait restée plus confidentielle. De là les chances d'intéresser un nouveau public, ce qui reste fondamental pour l'avenir, l’offre dépassant immensément la demande. Déjà, on constate que plus de jeunes compositeurs voyagent avec leur musique, de façon physique et virtuelle, et rendent la notion de marge obsolète, cela même en matière de styles musicaux comme si la page du monde n'avait plus ni centre ni marge, devenue territoire global où le nomade s'inscrit et «surfe» en quête du «spectaculaire», pour reprendre le terme employé par Alessandro Baricco dans son essai sur la mutation intitulé Les barbares:

Le spectaculaire est un mélange de fluidité, de rapidité, de sens de la synthèse et de technique qui provoque une accélération. On rebondit sur le spectaculaire. On jaillit. On reçoit de l'énergie au lieu d'en consommer ${ }^{4} \ldots$ 
Le rhizome va l'emporter sur la racine. Nul doute aussi que l'existence dans un environnement digital perpétuellement connecté provoquera cette mutation du cerveau et de la création musicale, qui s'avère déjà plus ludique et moins marquée par l'introversion, le fétichisme structurel et le drame des dissonances qui a pu caractériser le répertoire du $\mathrm{Xx}^{\mathrm{e}}$ siècle. Peut-être les (jeunes) mélomanes de l'avenir arriveront-ils pour leur part à écouter la musique non tonale en «suspendant le jugement tonal », si on me permet de paraphraser la définition que donne Milan Kundera du roman dans Les testaments trahis, à savoir qu'il est le «territoire où le jugement moral est suspendu ${ }^{5} » .$. Mais cette «suspension du jugement tonal» est-elle possible? Sinon maintenant, quand? Dans quelque âge d'or à venir, quand le Québec aura trouvé le siècle où ce sera lui, le «futur »? À moins que nous soyons déjà là?

Ma propre expérience de la marge, en bref? Après le Conservatoire et avoir longtemps cherché la «beauté » en composant, je sonde depuis quelques années les possibilités et la vérité de la «laideur», si ce terme veut encore dire quelque chose, comme consonance et dissonance... Je n'ai jamais été un pratiquant de l'extrême complexité ni de son envers simple. En fait, j’ai toujours résisté aux écoles de pensée esthétique demandant entière soumission. Néanmoins, dans la foulée d'une adolescence marquée par le surréalisme en poésie et en art, l'esprit dada a marqué ma production au milieu des années 1990, notamment dans le cadre de la création avec mon ami et collègue Silvio Palmieri, d'une exposition d'œuvres visuelles et d'un concert-spectacle à la Chapelle historique du BonPasteur ${ }^{6}$, précisément parce que ce mouvement rejetait tout dogme et prônait une "annihilation du passé» - de façon naïve mais combien rafraîchissante devant la sclérose inévitable des systèmes. Nous avions grandement besoin de sortir du cadre de la musique "pure et sérieuse »... De fait, la transgression fut nourrissante, mais je me suis ensuite rapidement mis en quête - sans le savoir alors! - d'une transgression de la transgression, pour reprendre une notion que j'ai ensuite découverte chez François Ricard dans sa postface de L'insoutenable légèreté de l'être de Kundera. La beauté pouvait être corrosive et la laideur envoûtante. En parallèle à la musique vocale et instrumentale en direct m’est revenu le désir de la musique électroacoustique (par besoin d'autonomie, pour pouvoir composer seul, dans la marge, sans interprètes, avec de nouveaux objets sonores) et de la musique mixte (en intégrant l'esprit d'un interprète parfois aussi improvisateur, pour réunir le meilleur de ces deux mondes). Tout change. Après 40 ans d'étude et de composition, la marge semble s'amenuiser et nous rapprocher tous du continent, dans le grand mariage inéluctable entre toutes les musiques qui va s'opérer au cours des siècles à venir, entre le «populaire» et le «savant», dont les frontières s'effacent aussi progressivement.

Y a-t-il un salut pour le compositeur, au-delà de quelque périodicité rythmique? Les milliards de personnes qui posent quotidiennement des écouteurs sur leurs oreilles et se soumettent aux pulsations de la pop le font sans qu'on les y oblige. Pourquoi? Trouvent-ils dans ce «centre global de la page» une catharsis et une satisfaction si grande? Les basses aux rythmes périodiques omniprésents sont-elles devenues les sirènes auxquelles l'être ne peut résister? Se pourrait-il que cette formidable adhésion s'explique aussi par le fait que les «états émotionnels» qui y sont déployés (et imposés par le marketing) sont plus lisibles et identifiables qu'ils ne le sont en musique de création, où la mouvance et l'ambiguïté harmoniques fondent le plus souvent des formes et des trajectoires intérieures aux résolutions non traditionnelles? À cet égard, se pourrait-il que le public «normal» non initié n'entende qu'ironie, complexité et «négativité » dans la musique de création, ce qui expliquerait son refus? La formule du concert de nouvelle musique, avec son enchaînement de plusieurs œuvres de courte durée et 
présenté une seule fois, est-elle optimale pour révéler les partitions? Pourquoi ne pas bisser les œuvres de façon systématique, en les alternant?

Pour le compositeur, se trouver dans un tel nonespace qui n'en finit plus de naître tient à la fois du rejet (indifférence du monde extérieur) et de la bénédiction (absence de diktats esthétiques). Cette affirmation demande nuances, car le monde réel et médiatique n'est pas entièrement et irrémédiablement fermé à la musique de création, et celle-ci peut d'ailleurs être asujettie de l'intérieur à des dogmes et des impositions idéologiques et esthétiques. Un des "problèmes", ce qui empêche l'adhésion, littéralement, du public normal et non musicien ou initié, c'est que les compositeurs n'ont pas d'image de marque, image idéalement cool de laquelle tous, semble-t-il, ont besoin pour s'extirper de l'anonymat. Une réelle incarnation du compositeur apparaît essentielle, sans quoi le médium reste trop flou et abstrait, avant même toute considération de la musique. Ici encore, les jeunes pallient rondement ce manque par l'autopromotion sur Facebook, où portraits, images et données abondent. Mais il faudra aussi un jour remettre en question la durée des œuvres commandées et donner la possibilité de composer des œuvres de longue durée, sans lesquelles les concerts prennent la forme de cocktails sonores. Sans revenir au canon wagnérien, il serait normal que des compositeurs puissent aussi composer des œuvres de 30,
60 ou même 90 minutes, pour dépasser l'impression et approfondir l'affirmation. Si le temps des «grands récits» est effectivement terminé, l'écriture des récits à venir est en marche. "Quand on est dans la merde jusqu'au cou, il ne reste qu'à chanter », disait Samuel Beckett. Alors, chantons encore.

1. Chronique sur l'actualité de la création musicale réalisée en collaboration avec les éditeurs du site Web <cettevilleetrange.org>, soit Paul Bazin, Bruno De Cat et Michel Gonneville. Les opinions exprimées dans cette publication n'engagent que leur auteur et ne représentent pas nécessairement celles de la revue Circuit. Des prolongements de cette collaboration sont publiés sur le site $<$ www.cettevilleetrange.org $>$. Les lecteurs sont invités à réagir en écrivant à info@ revuecircuit.ca ou à proposer une publication en écrivant à info@cettevilleetrange.org.

2. Ce titre fait référence à une citation de Samuel Beckett, avec cette différence que la "marge" y remplace le "merde" de l'original.

3. Milan Kundera (1993), Les testaments trahis: essai, Paris, Gallimard, p. 34.

4. Alessandro Baricco (2014), Les barbares: essai sur la mutation, traduit de l'italien par Françoise Brun et Vincent Raynaud, Paris, Gallimard, p. 169.

5. Kundera, 1993, p. 16.

6. Conçu et réalisé par Marc Hyland et Silvio Palmieri, "Dada Montréal " eut lieu à l'automne 1995. L'exposition d'art à la Chapelle historique du Bon-Pasteur regroupait entre autres des œuvres picturales de Beatrice Wood, Marcel Janco, John Cage, Charles Gagnon, Quentin Crisp, Marc Hyland, Silvio Palmieri, Gilles Groulx, Natalie Lord, Barbara Ulrich et Paul Lacerte. Le concert comprenait des œuvres d'Estelle Lemire, Jean Lesage, Anthony Rozankovic, Marie Pelletier, Ana Sokolovic, Katia Stockman, Marc Tremblay, un court-métrage de Hyland, une musique de Palmieri sur un film de Duchamp, la lecture du manifeste Dada et bien plus. 